\title{
Gauge-fixing independence of IR divergences in non-commutative $U(1)$, perturbative tachyonic instabilities and supersymmetry
}

\author{
F. Ruiz Ruiz \\ Departamento de Física Teórica I, Facultad de Ciencias Físicas, Universidad Complutense de Madrid, 28040 Madrid, Spain
}

Received 20 December 2000; accepted 17 January 2001

Editor: G.F. Giudice

\begin{abstract}
It is argued that the quadratic and linear non-commutative IR divergences that occur in $U(1)$ theory on non-commutative Minkowski space-time for small non-commutativity matrices $\theta^{\mu \nu}$ are gauge-fixing independent. This implies in particular that the perturbative tachyonic instability produced by the quadratic divergences of this type in the vacuum polarization tensor is not a gauge-fixing artifact. Supersymmetry can be introduced to remove from the renormalized Green functions at one loop, not only the non-logarithmic non-commutative IR divergences, but also all terms proportional to $\theta^{\mu \nu} p_{\nu}$. C 2001 Published by Elsevier Science B.V.
\end{abstract}

PACS: 11.15.-q; 11.30.Pb; 11.10.Gh

Keywords: Non-commutative $U(1)$ gauge theory; IR divergences; Gauge-fixing independence; Tachyonic instability

In perturbative $U(1)$ gauge theory on non-commutative space-time, one-loop 1PI Green functions are known to have a non-analytic behaviour for small values of the non-commutativity scale of space-time [1]. The corresponding singularities are called noncommutative IR divergences and occur in the nonplanar contributions of those Green functions whose planar parts are UV divergent [1]. This implies that one must expect quadratic and logarithmic non-commutative IR divergences in the vacuum polarization tensor, linear and logarithmic non-commutative IR singularities in the gauge tree-vertex, and

E-mail address: t63@aeneas.fis.ucm.es (F. Ruiz Ruiz). logarithmic non-commutative IR divergences in the gauge four-vertex [2]. In addition, and depending on the gauge choice, one should also expect non-commutative IR divergences in the ghost Green functions. The logarithmic non-commutative IR divergences in particular satisfy a very important duality relation with the UV divergences of the theory [1], in the sense that they can be very simply read from one another (see below for an explicit realization of this idea). By contrast, the quadratic and linear non-commutative IR divergences have no counterpart as UV singularities [2]. More importantly, their dependence on gauge-fixing is not known, and thus one is not able to elucidate whether they are true singularities or gauge-fixing artifacts. The purpose of this Letter is precisely to investi- 
gate their dependence on gauge-fixing. To do this, we will consider the theory in an arbitrary Lorentz gauge and compute the UV and non-commutative IR divergences at one loop. We will find that only the two and three-point functions of the gauge field, but not the ghost Green functions, contain quadratic and linear non-commutative IR divergences, that these do not depend on the gauge-fixing parameter and that they are equal to those previously obtained in the Feynman background gauge [3]. This suggests that quadratic and linear IR divergences are independent of gaugefixing. Having argued this, we will then show that they lead to a perturbative tachyonic instability for the theory. Finally, we will explicitly illustrate how to cancel them with supersymmetry [2].

Non-commutative Minkowski space-time is defined as the algebra generated by position operators $X^{\mu}$ satisfying commutation relations $\left[X^{\mu}, X^{\nu}\right]=$ $i \theta^{\mu \nu}$, with $\theta^{\mu \nu}$ an antisymmetric real matrix. Indices are raised and lowered with the Minkowski metric, for which we take $\eta_{\mu \nu}=\operatorname{diag}(+,-,-,-)$. On this space-time, a $U(1)$ gauge field is defined [4] as a real vector function $A^{\mu}(x)$ with field strength

$F_{\mu \nu}=\partial_{\mu} A_{\nu}-\partial_{\nu} A_{\mu}-i\left(A_{\mu} \star A_{\nu}-A_{\nu} \star A_{\mu}\right)$,

where the symbol $\star$ denotes the Moyal product of functions, i.e.,

$$
(f \star g)(x)=f(x) \exp \left(\frac{i}{2} \theta^{\mu \nu} \overleftarrow{\partial_{\mu}} \overrightarrow{\partial_{\nu}}\right) g(x) .
$$

The quantum $U(1)$ gauge theory can be defined through its Green functions, whose generating functional is formally given in an arbitrary Lorentz gauge by

$$
\begin{aligned}
Z[J, \bar{\eta}, \eta]= & \int[d A][d b][d \bar{c}][d c] \\
& \times \exp \left[i S+i \int d^{4} x(J A+\bar{\eta} c+\bar{c} \eta)\right] .
\end{aligned}
$$

Here $b$ is the auxiliary field imposing the Lorentz condition $\partial A=f$, with $f$ an arbitrary function of $x^{\mu}$, $\bar{c}$ and $c$ are ghost fields, and

$$
\begin{gathered}
S=\int d^{4} x\left(-\frac{1}{4 g^{2}} F^{\mu \nu} \star F_{\mu \nu}+\frac{\alpha}{2} g^{2} b \star b\right. \\
\left.-b \star \partial A+\bar{c} \star \partial_{\mu} D^{\mu} c\right)
\end{gathered}
$$

is the gauge-fixed classical action. As usual, $g$ denotes the coupling constant, $\alpha$ the gauge-fixing parameter, and $D_{\mu}$ the covariant derivative, whose action on a function $\omega(x)$ is $D_{\mu} \omega=\partial_{\mu} \omega-i\left(A_{\mu} \star \omega-\omega \star A_{\mu}\right)$. In what follows, to avoid running into inconsistencies with unitarity [5], we restrict ourselves to matrices $\theta^{\mu \nu}$ such that $\theta^{0 i}=0$ for $i=1,2,3$. This choice is called magnetic and ensures that the field theory exists as the zero slope limit of a string theory [6].

At one loop, the planar parts of only the vacuum polarization tensor $i \Pi_{\mu \nu}(p)$, the gauge threevertex $i \Gamma_{\mu_{1} \mu_{2} \mu_{3}}\left(p_{1}, p_{2}, p_{3}\right)$, the gauge four-vertex $i \Gamma_{\mu_{1} \mu_{2} \mu_{3} \mu_{4}}\left(p_{1}, p_{2}, p_{3}, p_{4}\right)$, the ghost self-energy $\Pi^{\bar{c} c}(p)$ and the ghost vertex $i \Gamma_{\mu}^{\bar{c} A c}\left(p_{1}, p_{2}, p_{3}\right)$ are UV divergent. Therefore, one should expect noncommutative IR singularities in only these Green functions. To regularize the theory, we use dimensional regularization with $D=4+2 \epsilon$. When sending $\epsilon \rightarrow 0$, two types of terms appear: $1 / \epsilon$ pole terms and finite terms. The pole terms, being divergent, we keep them. The finite terms, as functions of $\theta^{\mu \nu}$, develop singularities for $\theta^{\mu \nu} \rightarrow 0$. These singularities are our main interest here. We have computed the double limit $\lim _{\theta \rightarrow 0} \lim _{\epsilon \rightarrow 0}$ of these five Green functions and obtained, modulo finite contributions:

$$
\begin{aligned}
i \Pi_{\mu \nu}(p)= & \frac{2 i}{\pi^{2}} \frac{\tilde{p}_{\mu} \tilde{p}_{\nu}}{(p \circ p)^{2}}-\frac{i}{16 \pi^{2}}\left(\frac{13}{3}-\alpha\right) \\
& \times\left[\frac{1}{\epsilon}-\ln \left(p^{2} p \circ p\right)\right]\left(p^{2} \eta_{\mu \nu}-p_{\mu} p_{\nu}\right),
\end{aligned}
$$

$i \Gamma_{\mu_{1} \mu_{2} \mu_{3}}\left(p_{1}, p_{2}, p_{3}\right)$

$$
\begin{aligned}
=\frac{2}{\pi^{2}} & \cos \left(\frac{p_{1} \wedge p_{2}}{2}\right) \sum_{i=1}^{3} \frac{\left(\tilde{p}_{i}\right)_{\mu_{1}}\left(\tilde{p}_{i}\right)_{\mu_{2}}\left(\tilde{p}_{i}\right)_{\mu_{3}}}{\left(p_{i} \circ p_{i}\right)^{2}} \\
+ & \frac{1}{16 \pi^{2}} \sin \left(\frac{p_{1} \wedge p_{2}}{2}\right)\left(\frac{17}{3}-3 \alpha\right) \\
\times & {\left[\frac{1}{\epsilon}-\frac{1}{3} \sum_{i=1}^{3} \ln \left(p_{i} \circ p_{i}\right)\right] } \\
\times & {\left[\eta_{\mu_{1} \mu_{2}}\left(p_{1}-p_{2}\right)_{\mu_{3}}+\eta_{\mu_{2} \mu_{3}}\left(p_{2}-p_{3}\right)_{\mu_{1}}\right.} \\
& \left.+\eta_{\mu_{3} \mu_{1}}\left(p_{3}-p_{1}\right)_{\mu_{2}}\right],
\end{aligned}
$$

$i \Gamma_{\mu_{1} \mu_{2} \mu_{3} \mu_{4}}\left(p_{1}, p_{2}, p_{3}, p_{4}\right)$

$$
=\frac{i}{16 \pi^{2}}\left[4\left(\eta_{\mu_{1} \mu_{3}} \eta_{\mu_{2} \mu_{4}}-\eta_{\mu_{1} \mu_{4}} \eta_{\mu_{2} \mu_{3}}\right)\right.
$$




$$
\begin{aligned}
& \times \sin \left(\frac{p_{1} \wedge p_{2}}{2}\right) \sin \left(\frac{p_{3} \wedge p_{4}}{2}\right) f_{\epsilon}\left(\tilde{p}_{1}, \tilde{p}_{2}\right) \\
& +4\left(\eta_{\mu_{1} \mu_{2}} \eta_{\mu_{3} \mu_{4}}-\eta_{\mu_{1} \mu_{4}} \eta_{\mu_{2} \mu_{3}}\right) \\
& \times \sin \left(\frac{p_{1} \wedge p_{3}}{2}\right) \sin \left(\frac{p_{2} \wedge p_{4}}{2}\right) f_{\epsilon}\left(\tilde{p}_{1}, \tilde{p}_{3}\right) \\
& +4\left(\eta_{\mu_{1} \mu_{2}} \eta_{\mu_{3} \mu_{4}}-\eta_{\mu_{1} \mu_{3}} \eta_{\mu_{2} \mu_{4}}\right) \\
& \left.\times \sin \left(\frac{p_{1} \wedge p_{4}}{2}\right) \sin \left(\frac{p_{2} \wedge p_{3}}{2}\right) f_{\epsilon}\left(\tilde{p}_{1}, \tilde{p}_{4}\right)\right] \text {, } \\
& i \Pi^{\bar{c} c}(p)=\frac{i}{16 \pi^{2}} \frac{3-\alpha}{2}\left[\frac{1}{\epsilon}-\ln (p \circ p)\right] p^{2}, \\
& i \Gamma_{\mu}^{\bar{c} A c}\left(p_{1}, p_{2}, p_{3}\right) \\
& =-\frac{\alpha}{16 \pi^{2}} \sin \left(\frac{p_{2} \wedge p_{3}}{2}\right) \\
& \times\left[\frac{1}{\epsilon}+\ln \left(p_{1} \circ p_{1}\right)-\ln \left(p_{2} \circ p_{2}\right)\right. \\
& \left.-\ln \left(p_{3} \circ p_{3}\right)\right]\left(p_{1}\right)_{\mu} \text {. }
\end{aligned}
$$

Here $\tilde{p}^{\mu}, p_{i} \wedge p_{j}$ and $p \circ p$ denote

$\tilde{p}^{\mu}=\theta^{\mu \nu} p_{\nu}, \quad p \wedge q=\theta^{\mu \nu} p_{\mu} q_{\nu}$,

$p \circ p=-\theta^{\mu \nu} \theta_{\mu}{ }^{\tau} p_{\nu} p_{\tau}$,

and the function $f_{\epsilon}\left(\tilde{p}_{j}, \tilde{p}_{k}\right)$ is given by

$$
\begin{aligned}
f_{\epsilon}\left(\tilde{p}_{j}, \tilde{p}_{k}\right) & \\
= & \frac{1}{\epsilon}\left(\frac{4}{3}-2 \alpha\right) \\
& +\frac{1}{8}\left[(\alpha+3)(\alpha-1)+\frac{31}{3}\right] \sum_{i=1}^{4} \ln \left(p_{i} \circ p_{i}\right) \\
& -\frac{1}{2}\left[9+(1-\alpha)^{2}\right] \ln \left[\left(p_{j}+p_{k}\right) \circ\left(p_{j}+p_{k}\right)\right] .
\end{aligned}
$$

In the ghost vertex $i \Gamma_{\mu}^{\bar{c} A c}\left(p_{1}, p_{2}, p_{3}\right), p_{1}^{\mu}, p_{2}^{\mu}$ and $p_{3}^{\mu}$ are the momenta of the incoming ghost, the gauge field and the outgoing ghost, respectively. The $1 / \epsilon$ pole terms in these equations were first calculated in Ref. [7], give for the one-loop beta function $\beta_{1}=$ $-11 / 3[7,8]$ and are subtracted by adding UV counterterms. This UV renormalization procedure leaves the non-commutative IR divergences unchanged, a feature observed in all field theories considered so far [9].

We observe that the first term in the vacuum polarization tensor (2) diverges quadratically for $\theta^{\mu \nu} \rightarrow$
0 and that the first term in the gauge three-vertex (3) does it linearly. More importantly, their coefficients do not depend on the gauge-fixing parameter $\alpha$ and are equal to those obtained in the Feynman background gauge [3]. We would like to emphasize that this $\alpha$-independence is not trivial. To illustrate this, suffice to say that the Feynman diagrams that contribute to, e.g., the gauge three-vertex give linear non-commutative IR divergent contributions of type $\cos \left(\frac{1}{2} p_{1} \wedge p_{2}\right) \eta_{\mu_{1} \mu_{2}}\left[\left(\tilde{p}_{i}\right)_{\mu_{3}} /\left(p_{i} \circ p_{i}\right)\right]$ and $\cos \left(\frac{1}{2} p_{1} \wedge p_{2}\right)\left[\left(\tilde{p}_{i}\right)_{\mu_{1}}\left(\tilde{p}_{i}\right)_{\mu_{2}}\left(\tilde{p}_{i}\right)_{\mu_{3}} /\left(p_{i} \circ p_{i}\right)^{2}\right]$ whose coefficients depend on $\alpha$, and that only after summation over diagrams these contributions cancel except for the first term in Eq. (3). We also note that the ghost self-energy does not have linear non-commutative IR divergences, even though on general grounds it is susceptible to them. All this suggests that the quadratic and linear non-commutative IR divergences in the theory at one loop are gauge-fixing independent. By contrast, the logarithmic terms $\ln (p \circ p)$ depend on $\alpha$ and are in complete accordance with UV/IR duality. Indeed, in the non-commutative IR momentum domain $\left|\tilde{p}_{i}\right| \sim \theta \Lambda_{\mathrm{IR}} \rightarrow 0$, the terms in a Green function with logarithmic dependence on $\theta^{\mu \nu}$ can be obtained from the pole terms in the same Green function through the identifications $1 / \epsilon \leftrightarrow \ln \Lambda_{\mathrm{UV}}^{2}, \Lambda_{\mathrm{UV}} \leftrightarrow 1 / \theta \Lambda_{\mathrm{IR}}$.

Now that we have argued that the quadratic noncommutative IR divergences are independent of the choice of gauge, we discuss their relevance for perturbative tachyonic instability. We use the notation $p^{\mu}=(E, \vec{p})$ and consider momenta in the domain $g^{2} /(p \circ p)|\vec{p}|^{2} \ll 1$, where perturbation theory is valid. In this domain, the leading behaviour of the renormalized vacuum polarization tensor in any admissible renormalization scheme is given by

$$
\begin{aligned}
i \Pi_{\mu \nu}^{\mathrm{ren}}(p)= & -\frac{i}{g^{2}}\left(p^{2} \eta_{\mu \nu}-p_{\mu} p_{\nu}\right)+\frac{i a}{\pi^{2}} \frac{\tilde{p}_{\mu} \tilde{p}_{v}}{(p \circ p)^{2}} \\
& + \text { subleading contributions, }
\end{aligned}
$$

where, according to Eq. (2), $a=2$. For a "photon" of momentum $p^{\mu}$ polarized in the direction of $\tilde{p}^{\mu}$, with plane wave field $f(p) \tilde{p}^{\mu} e^{i p x}$, the dispersion relation takes the form

$E^{2}=|\vec{p}|^{2}-\frac{a}{\pi^{2}} \frac{g^{2}}{p \circ p}$.

Since $a=2$ and $p \circ p$ is positive, this gives rise to a perturbative tachyonic instability. Note that the ex- 
istence of this instability depends on the sign of $a$; had $a$ been negative, there would not be such instability. Instabilities of this type have also been found in scalar theories on non-commutative space-times [10]. The quadratic and linear non-commutative IR divergences under consideration are also a source of problems for UV renormalizability at higher loops, since a one-loop non-commutative IR divergence nested in a two-loop diagram produces IR non-integrable singularities which make renormalization at two loops unlikely. All in all, one would like to find a way to cancel these non-commutative IR divergences. It has been argued in Ref. [2] that in the Feynman Lorentz gauge this can be done by introducing supersymmetry. The gauge-fixing independence of the divergences under consideration, together with the observation that the coupling of fermions to the gauge field is through the covariant derivative and thus does not depend on gauge-fixing, makes the cancellation picture consistent. Let us explicitly realize this cancellation mechanism for $N=1$ supersymmetry.

The coupling of a Majorana spinor $\lambda$ to the gauge field $A^{\mu}$ adds a term $-\frac{1}{2 g^{2}} \bar{\lambda} \star \not D \lambda$ to the classical action. Since the field $\lambda$ must belong to the same supermultiplet as the gauge field, it must transform according to the adjoint representation and thus for the covariant derivative we must take $D_{\mu} \lambda=\partial_{\mu} \lambda-$ $i\left(A_{\mu} \star \lambda-\lambda \star A_{\mu}\right)$. At one loop, this coupling gives additional contributions to the two, three and fourpoint 1PI functions of the gauge field. For brevity, we only display here the first two:

$$
\begin{aligned}
\Delta\left[i \Pi_{\mu \nu}(p)\right]=- & \frac{2 i}{\pi^{2}} \frac{\tilde{p}_{\mu} \tilde{p}_{\nu}}{(p \circ p)^{2}} \\
- & \frac{i}{16 \pi^{2}} \frac{4}{3}\left[-\frac{1}{\epsilon}+\ln \left(p^{2} p \circ p\right)\right] \\
& \times\left(p^{2} \eta_{\mu \nu}-p_{\mu} p_{\nu}\right)
\end{aligned}
$$

$$
\begin{aligned}
\Delta\left[i \Gamma_{\mu_{1} \mu_{2} \mu_{3}}\left(p_{1}, p_{2}, p_{3}\right)\right] \\
=-\frac{2}{\pi^{2}} \cos \left(\frac{p_{1} \wedge p_{2}}{2}\right) \sum_{i=1}^{3} \frac{\left(\tilde{p}_{i}\right)_{\mu_{1}}\left(\tilde{p}_{i}\right)_{\mu_{2}}\left(\tilde{p}_{i}\right)_{\mu_{3}}}{\left(p_{i} \circ p_{i}\right)^{2}} \\
+\frac{1}{16 \pi^{2}} \sin \left(\frac{p_{1} \wedge p_{2}}{2}\right) \\
\quad \times \frac{8}{3}\left[-\frac{1}{\epsilon}+\frac{1}{3} \sum_{i=1}^{3} \ln \left(p_{i} \circ p_{i}\right)\right]
\end{aligned}
$$

$$
\begin{aligned}
\times & {\left[\eta_{\mu_{1} \mu_{2}}\left(p_{1}-p_{2}\right)_{\mu_{3}}+\eta_{\mu_{2} \mu_{3}}\left(p_{2}-p_{3}\right)_{\mu_{1}}\right.} \\
& \left.+\eta_{\mu_{3} \mu_{1}}\left(p_{3}-p_{1}\right)_{\mu_{2}}\right] .
\end{aligned}
$$

We see that indeed these two contributions cancel the quadratic and linear non-commutative IR divergences in Eqs. (2) and (3). Although we are not displaying partial results here, we mention that the entire contribution to the vacuum polarization tensor proportional to $\tilde{p}_{\mu} \tilde{p}_{\nu}$, and not only its divergent part for $\theta^{\mu \nu} \rightarrow 0$, is canceled by the contribution proportional to $\tilde{p}_{\mu} \tilde{p}_{v}$ from the Feynman diagram with Majorana fermions flowing along the loop. Hence the vacuum polarization tensor for the supersymmetric theory is, for arbitrary values of $\theta^{\mu \nu}$, proportional to $p^{2} \eta_{\mu \nu}-p_{\mu} p_{\nu}$. This implies that there is no momentum region for which a tachyonic instability could arise at one loop. Analogously, all terms in the gauge three-vertex proportional to any $\tilde{p}_{i}^{\mu}(i=1,2,3)$, and not only their divergent parts for $\theta^{\mu \nu} \rightarrow 0$, are canceled by supersymmetry. All this does not guarantee, however, that the supersymmetric theory is free of non-logarithmic noncommutative IR divergences at one loop, since linear non-commutative IR singularities may still occur in the Majorana fermion self-energy $\Sigma(p)$. To check if this is so we have calculated $\Sigma(p)$ and obtained that it has no contribution at all proportional to $\gamma^{\mu} \tilde{p}_{\mu}$ and that in the double limit of interest it reduces to

$$
\Sigma(p)=-\frac{\alpha}{16 \pi^{2}}\left[\frac{1}{\epsilon}-\ln \left(p^{2} p \circ p\right)\right] p,
$$

which only contains logarithmic non-commutative IR divergences. In summary, in the supersymmetric theory all non-commutative IR divergences are logarithmic and the tensor structures that enter the one-loop 1PI Green functions are the same as for the conventional commutative case, thus increasing the expectations for renormalizability at higher loops. Note finally that supersymmetry modifies the beta function, which now is $\beta_{1}=-3$.

We conclude summarizing our results. We have argued that the quadratic and linear non-commutative IR divergences in $U(1)$ gauge theory on non-commutative space-time are gauge-fixing independent. From the point of view of local perturbative quantum field theory, the presence of non-logarithmic non-commutative IR divergences is problematic. Indeed, a renormalized one-loop 1PI Green function with divergences of this type, when nested in a two-loop diagram, gives rise for 
small $\theta^{\mu \nu}$ to non-integrable singularities, thus making unlikely UV renormalization at higher loops. In addition, the quadratic non-commutative IR divergences in the vacuum polarization tensor yield, for polarizations parallel to the non-commutative directions, a tachyonic dispersion relation which introduces a perturbative tachyonic instability. One way to remove these non-commutative IR divergences and the problems they bring along is to introduce supersymmetry, since 1PI Feynman diagrams with Majorana fermions flowing along the loop provide the necessary contributions to cancel the quadratic and linear non-commutative IR divergences in the Green functions of the gauge field. Further, since the self-energy of the Majorana fermion does not contain divergences of this type, the supersymmetric theory is free of quadratic and linear noncommutative IR divergences. Thus in a superfield approach, one expects to only find UV and logarithmic non-commutative IR divergences [11].

\section{Acknowledgements}

The author is grateful C.P. Martín for many conversations and discussions, and to CICyT, Spain, for financial support through grant No. PB98-0842.

\section{References}

[1] S. Minwalla, M.V. Raamsdonk, N. Seiberg, JHEP 0002 (2000) 020.

[2] A. Matusis, L. Susskind, N. Toumbas, The IR/UV connection in the non-commutative gauge theories, hep-th/0002075.

[3] C.P. Martin, F. Ruiz Ruiz, Paramagnetic dominance, the sign of he beta function and UV/IR mixing in non-commutative $U(1)$, hep-th/0007131, to appear in Nucl. Phys. B.

[4] A. Connes, Non-commutative geometry, Academic Press, New York, 1994;

G. Landi, in: An introduction to non-commutative spaces and their geometries, Springer Lecture Notes in Physics, Vol. 51, Springer-Verlag, Berlin, 1997.

[5] J. Gomis, T. Mehen, Nucl. Phys. B 591 (2000) 265.

[6] N. Seiberg, L. Susskind, N. Toumbas, JHEP 0006 (2000) 021; J.L.F. Barbón, E. Rabinovici, Phys. Lett. B 486 (2000) 202.

[7] C.P. Martín, D. Sánchez-Ruiz, Phys. Rev. Lett. 83 (1999) 476.

[8] T. Krajewski, R. Wulkenhaar, J. Mod. Phys. A 15 (2000) 1011.

[9] M.M. Sheikh-Jabbari, JHEP 9906 (1999) 015; M. Hayakawa, Phys. Lett. B 478 (2000) 394;

I.Ya. Aref'eva, D.M. Belov, A.S. Koshelev, O.A. Rytchkov, UV/IR mixing for noncommutative complex scalar field theory, II (interaction with gauge fields), hep-th/0003176;

A. Armoni, Nucl. Phys. B 593 (2001) 229.

[10] J. Gomis, K. Landsteiner, E. López, Phys. Rev. D 62 (2000) 105006;

K. Landsteiner, E. López, M.H.G. Tytgat, JHEP 0009 (2000) 027;

J. Gomis, T. Mehen, M.B. Wise, JHEP 0008 (2000) 029.

[11] D. Zanon, Noncommutative $\mathcal{N}=1,2$ super $U(N)$ YangMills: UV/IR mixing and effective action results at one loop, hep-th/0012009. 